\title{
USE: a concept-based recommendation system to support creative search
}

\author{
J. Sousa Lopes, S. Alvarez-Napagao, J. Vázquez-Salceda \\ KEMLg - Knowledge Engineering and Machine Learning Group \\ UPC - Universitat Politècnica de Catalunya \\ Barcelona, Spain \\ \{jsousalopes, salvarez,jvazquez\}@lsi.upc.edu
}

\begin{abstract}
Semiotics is a field on which research in Computer Science methodologies has focused, mainly concerning Syntax and Semantics. These methodologies, however, are lacking some flexibility for the continuously evolving web community, in which the knowledge is classified with tags rather than with ontologies. In this paper we propose a system for the recommendation of tagged pictures obtained from the Web. The system, driven by user feedback, executes an abductive reasoning (based on WordNet synset semantic relations) that is able to iteratively lead to new concepts which progressively represent the cognitive creative user state.
\end{abstract}

Keywords-semiotics; wordnet; tags; abduction; inference; semantics;

\section{INTRODUCTION}

Humans live in an exciting and dynamic environment that allows them to be prolific and creative. But there are human boundaries especially in terms of cognitive and mental power. For instance, it is currently acknowledged that in short-term memory there are only 4-7 conceptual chunks we can grasp [1]. We frequently have difficulty visualizing a set of vectors in three dimensions, let alone doing so in higher dimensions. With the fast growing technology in the computer industry, we have become familiarized with using machines as our computational and cognitive prostheses. That is, we rely on their power to help us accomplish tasks. Although there exist many tools that drive users on the creative process by providing the tools that shorten the effort between an idea generation and its implementation, there is a lack of applications that work directly on idea generation by means of truly recognizing and working on the cognitive symbolic representation.

Nowadays communication professionals, particularly those who work on visual communication and branding design, do not have any specialized tool to work on an individual brainstorming. Creative search is usually about finding innovative relationships between concepts. In a way, being creative is, from our perspective, to discover, to transfer, to modify and to assign meaning. More and more the search for inspiration is being converted into a common free navigation over the Internet, relating concepts and matching associations in the context of a non-cooperative meaning retrieval process.

In this context, recommendation systems can be useful in building innovative tools that lead those professionals in their creative searches. The emergence of recommendation systems was fostered by the user need to deal with internet information overload. Such systems are a means to provide personalized recommendations, content, and services to them. They have been studied and applied in several domains such as Web content, music, movies recommendation. In recommender systems users rate items they like and such ratings are stored and used by the systems to make further recommendation.

In literature, recommendation systems have been classified into content-based, collaborative filtering and hybrid systems [2]. Content-based systems recommend the user by means of a utility function which computes the degree of similarity of new items w.r.t. the previously user rated ones and especially deal with text-based recommendation (e.g., Web pages). The recommendation process is based on keywords and some heuristics or Bayesian classifiers [3]. Collaborative recommendation systems are based on ratings computed by the utility function taking into account the rate of other users to the same items [4]. Both approaches deal moreover with absolute value prediction of non rated items. The prediction is estimated in many different ways using methods from machine learning such as clustering, decision trees and artificial neural networks, approximation theory, and various domain-specific heuristics. Several problems have been identified for such approaches, such as limited content analysis, new user, new items, overspecialization, and sparsity [5]. Although some of these problems can be overcome by hybrid approaches [6], others such as the comprehensive understanding/building of the users profiles/behaviors and non-intrusiveness [5] still remain unsolved.

We have designed a concept-based recommendation system that uses the semantic relations between tags on images to formulate some contextual hypotheses that are expressed by new words. The proposed system helps creative professionals to find a concept idea with less effort on a constructive and interactive way. The suggested online application is built on top of a logical abduction model which, by applying a continuous interaction-mediated proof and rebuttal, induces some new user semantic relations between concepts. 
Tags are metadata pieces encoded in the form of keywords or terms which try to identify a single information item. This cognitive process has a wide degree of freedom, and only requires from the user to write down a list of related but potentially arbitrary keywords [7]. It can be argued that this "excessive" freedom required by the tagging process makes it difficult, if not impossible, to properly classify and give semantics to the concepts and relationships defined by the tags. However, in the last years research on tagging has been growing and solutions to this problem are currently being addressed [8].

The semantic model and support tool we use to relate those tags and to set a human-driven cognitive modulation is WordNet, an online lexical database designed for use under program control, with more than 166,000 word form and sense pairs.

The rest of the paper presents our proposed recommender system. Section II presents the system, how interaction is driven and a perspective on user's interface; in Section III we model USE, presenting how interaction cycles are processed; in Section IV we unveil the abductive logic used; in Section $\mathrm{V}$ we describe some of the related work and finally, in Section VI, we present some future lines of research and the conclusions.

\section{ThE USE SySTEM}

Mental processing in humans is activated through symbols which have been developed to identify and describe the sensations caused by the integration of various stimuli impacting upon the senses. Such sensations help the individual to organize these various sense perceptions into a single comprehension. These comprehensions are then subject to the development or adoption of symbolic representations which not only allow us to consciously comprehend the idea caused by the sensation, but to communicate it as well. In modern societies, as we become less aware of nonverbal or intuitive sensations, we increasingly use words or symbolic language in our thought processes. Therefore when we want to represent an idea an amount of symbols are used as internal conventions to spare and catalog those stimuli.

In this context, we introduce USE (Uplift Seek Engine), a tool for the improvement of the individual brainstorm process for the search of images based on the tagging information attached to them. This is accomplished by receiving the users' input on the images' conceptual accuracy as well as the description tags assigned to it. Beside this cycle interaction, the system also uses the generated history of the semantic relationships established between the concepts and the tags.

By its conception, USE is a self-brainstorming engine that pursuits the attempt to establish a systems-semiotic framework to help creativity in the design process, where the design process is considered to have the cognitive process as its basis, identified as a dynamic relationship between abstract concepts mapped over a sequence of selected signs.

Concept navigation, represented as a ow, allows the users for some different approaches whether the state of the work is: on the problem definition (including problem analysis, redefinition, and all aspects associated with clearly defining the problem); on the idea generation (related to the divergent process of coming up with ideas); or on the idea selection (as the convergent process of reducing all the many ideas into realistic solutions). The identification of the exact conjuncture is based on an abductive reasoning which attempts to generate the best explanation describing the users' acknowledged and disproved concepts that are related with the ideation cognitive process.

We use WordNet as a support tool for the retrieval of semantic knowledge associated to tags. WordNet [9] is a large browseable lexical database, available in several languages, which groups synsets expressing a distinct concept. We use tags related to pictures because they represent a description based on a perceived linguistic interpretation, meaning that this metadata associated with each image is already itself an abductive inference processed by the users that wrote those tags. This inference is processed by unveiled sign processing (semiosis) means.

The symmetry between the already processed semiosis on the description and the newest interpretation, on a creative process, by our system differs from any text-based one in that it facilitates an endless chain of symbolic relations. We can say that our semiotic machine is also a dialectic interpreter and actor that employs the results of user interaction in order to search for new concepts which are semantically related to the user choices. The application has two main sections, presented after the user has been identified on a login screen:

- Start idea and ideation cloud: in this screen the user starts a new creative process by writing a word that best fits the main concept and an unlimited number of other words chosen by their nearness to the first concept. Users can also identify some concepts that they do not want to be closely related to the main one;

- Ideation machine: in this screen the results of the successive processed abductions are presented by showing some images for the users' appreciation. The endless interaction process in this section expresses not just the confirmation or refutability of the abduction logic hypothesis about some particular synset but also, as each image has more than one tag, for all the description words, and their related synsets. If there are two or more coincident abductions that are proven to be true, by means of user acceptance, we induce a generalized new rule that is recorded on the graph. Notice that these new relations are user specific and therefore they drive the semantic mock-up for a more optimal cognitive semantic relationship representation. 


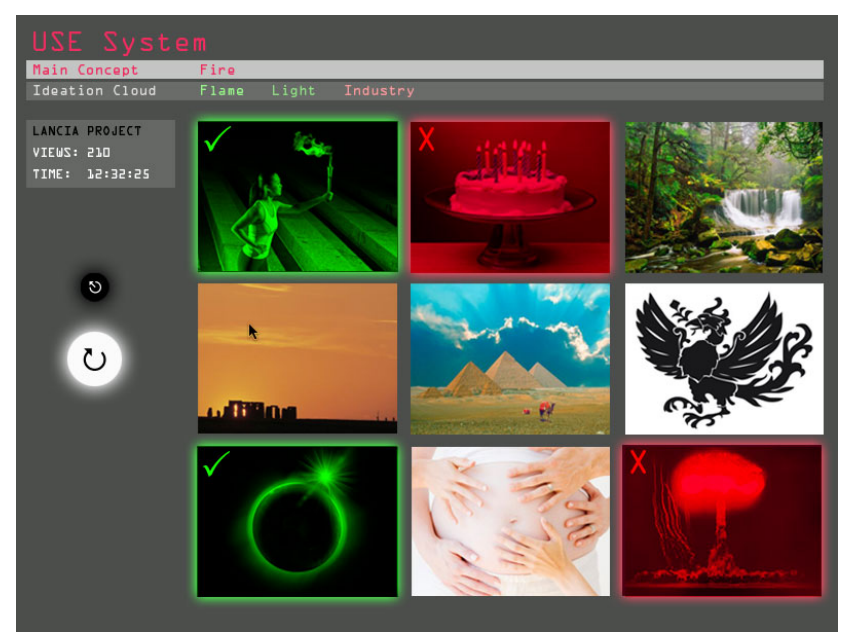

Figure 1. USE's screenshot of the ideation machine

In USE, our target users are highly technology-dependent and with a particular sensibility to applications that have no objectively defined purpose. Hence they prefer to spend much of their time in virtual playgrounds or on open-mind activities. The essence of the creative process is specifically the abstraction of the duty of finding distinctive ways to communicate the target values.

Because there is no deep enough research on concentrating and maximizing the efforts of these users, we consider USE a novel tool with a scalable architecture that can be applied in other domains that deal with cognitive processes and conceptual knowledge generation. USE is, in conclusion, a tool raised from the need to find a new idea within the minimum amount of time and in an intuitive and relaxing way.

\section{ABDUCTION}

Abduction, as opposed to deduction and induction ${ }^{1}$, is based on the inference of $\phi$ (explanans) from knowledge of the rule $\phi \rightarrow \psi$ and the observation $\psi$ (explanandum). This means that abduction is not an analytic form of inference, but rather based on the Affirming the Consequent fallacy. Like induction, abduction is defeasible: the arrival of new observations might invalidate prior abductive inferences.

The conditions which define when a fact $\phi$ qualifies as a valid abductive explanation for an observed fact $\psi$, with a background theory $\Theta$, are [10]:

- $\Theta \cup \phi \models \psi$

- $\Theta \cup \phi \not \models \perp$

- $\Theta \not \models \psi$

- $\phi \not \forall \psi$

Our main objective is to show how logic conclusions inferred via abductions can be useful to construct a knowledge

\footnotetext{
${ }^{1}$ Deduction is based on the modus ponens syllogism $(\{\phi, \phi \rightarrow \psi\} \models \psi)$, while induction is based on the inference of $\phi \rightarrow \psi$ as a rule from the observation of $\phi$ followed by $\psi$
}

model that can be reused in a knowledge retrieval process. We have already applied the concept of reasoning-driven abduction in a model of a real industrial process, and a trace of the abduction inferences can be found at $[11]^{2}$.

The formal logic used in our proposal is $\mathbf{L} \mathbf{A}^{r}$, presented in [13]. $\mathbf{L} \mathbf{A}^{r}$ is a logic based on Classical Logic with a non-monotonic dynamical process in which deductive steps are combined with abductive steps. Abductive steps may be withdrawn if, via deductive steps, its negation is derived.

This logic represents abductive steps as formulas of the form:

$$
B(\beta),(\forall \alpha)(A(\alpha) \supset B(\alpha)) / A(\beta)
$$

In this formula we identify three components: $B(\beta)$, which is the fact to be explained (explanans), $(\forall \alpha)(A(\alpha) \supset$ $B(\alpha)$ ), which is the deductive rule, and $A(\beta)$, which is the explanation (explananda).

\section{Modelling USE}

In WordNet [9], the vocabulary is seen as a set $W$ of pairs $(f, s)$ where $f$ is a word and $s$ is a sense of that word. From this definition, we will define $\Phi_{f}$ as the set of possible senses for a word $f$ in $W$, and $\Psi_{s}$ as the set of the possible words for a sense $s$ in $W$. For example,

$$
\begin{gathered}
\Phi_{\text {fire }}=\{\text { fire } \# 1, \ldots, \text { fire } \# 9, \text { ardor } \# 3\} \\
\Psi_{\text {fire\# } 3}=\{\text { fire }, \text { flame }, \text { flaming }\}
\end{gathered}
$$

WordNet defines a set of semantic relations between these senses. From this set, we will focus on the two relations that bidirectionally describe the $I s-A$ hierarchy: hypernyms $\left(s_{i}\right)$, and hyponym $\left(s_{i}\right)$. For example,

$$
\text { hypernyms }(\text { fire } \# 3)=\{\text { combustion\# } 1\}
$$

hyponyms $($ fire\#3 $)=\{$ blaze\# 1, flare\#2, ignition $\# 1\}$

Each retrieved image $i$ includes a set of tags defined by the user that uploaded it: $T_{i}=\left\{t_{1}, \ldots, t_{N}\right\}$. We will consider as words $w_{i}=t_{i}$ those tags that have at least one sense in the vocabulary $W$, that is, $\exists\left(t_{i}, s\right) \in W$. For the purpose of our system, we will define the image tagging $I_{i}$ of the

\footnotetext{
${ }^{2}$ This industrial process use case showed the viability of abduction in those cases where the agent has access to limited observations, because of, for example, a sensor fault, and it needs to maintain a certain degree of definition about the world state and therefore enduring discontinued operation. The knowledge extracted about correlations between distant entities can also be used to develop new production strategies based on hypothesis revision and test experiments suggested by the uncertainness of abduction explanation. For that reason, both creative and operation goal issues can be accomplished with abductive reasoning [12].
} 


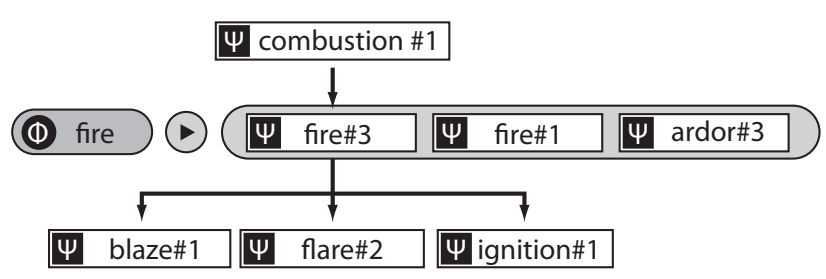

Figure 2. Synset, hypernyms and hyponyms of fire

image $i$ as the set of senses belonging to the set of valid words found in the tags:

$$
I_{i}=\bigcup_{\left(w_{i}, s_{j}\right) \in W, \forall w_{i} \in T_{i}} s_{j}
$$

The interaction of the user is defined as the succession of steps $k$ in which the user marks individually the images that are retrieved. Thus, for each image there are three possible actions: mark as positive, mark as negative, and leave unmarked.

In our system, we treat each of the steps $k$ as a particular independent case. In each of them, a set of tags with a certain truth value are produced. In order to incorporate them into our abduction system, we add them to the proof of the abductive system in the form of predicates $P(x)$, where $P$ is the tag, and $x$ is the variable representing the specific step. Therefore, these predicates represent the desirability of that specific tag in the context of a particular user interaction.

The result of this interaction will be:

- For each picture marked as positive, all of their tags will be added as predicates.

- For each picture marked as negative, all of their tags that are not in any of the pictures marked as positive will be added as negated predicates, that is, with a truth value of false.

- For each picture left unmarked, nothing is done.

In order to apply the abductive process based on $\mathbf{L} \mathbf{A}^{r}$ to $U S E$, we employ a mapping from these components to the basic elements of USE.

The facts in our system are the predicates representing the user interaction. The rules are the semantic relations between words, in our case the ones representing the $I s$ - $A$ hierarchy: hypernyms and hyponyms. Considering a sense $s$, each of its hypernyms $s^{\prime}$ is a generalization, and thus $s \rightarrow s^{\prime}$, and each of its hyponyms $s^{\prime \prime}$ is an specialization, and thus $s^{\prime \prime} \rightarrow s$ :

$$
\begin{aligned}
& s^{\prime} \in \operatorname{hypernym}(s) \Rightarrow(\forall \alpha)\left(s(\alpha) \supset s^{\prime}(\alpha) \wedge \neg s^{\prime}(\alpha) \supset \neg s(\alpha)\right) \\
& s^{\prime \prime} \in \operatorname{hyponym}(s) \Rightarrow(\forall \alpha)\left(s^{\prime \prime}(\alpha) \supset s(\alpha) \wedge \neg s(\alpha) \supset \neg s^{\prime \prime}(\alpha)\right)
\end{aligned}
$$

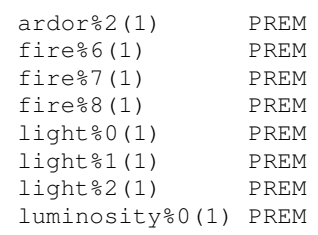

Figure 3. Premises (part)

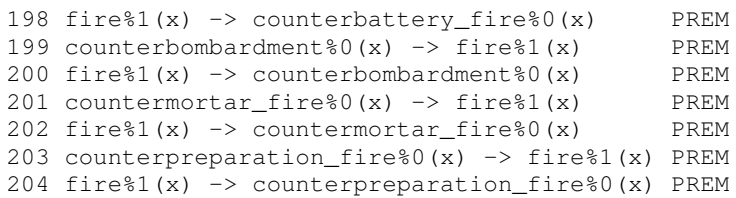

Figure 4. Rules (part)

327 flare $\%(1) \quad \operatorname{RC} 263,2$ \{ffire $\%$ (1), flare $\% 1(1)\}\}$

328 ignition\%0(1) RC 265,2 \{\{fire\%2(1), ignition\%0(1)\}\}

330 cookfire\%0(1) RC 269,3 \{ffire\%3(1), cookfire\%0(1)\}

331 zeal\%1(1) RC 271,5 \{\{ardor\%2(1), zeal\%1(1)\}

Figure 5. Abductions (part)

All these rules are implicitly derived from the WordNet database at runtime and added to the proof, as needed. ${ }^{3}$ At the beginning of the process, the proof is filled by the set of premises representing the senses of the word inputs of the user and the rules derived from the senses of the main concept.

After each step of user interaction, an abduction process is executed in the proof and repeated until no new facts are added. Every time a contradiction appears in the proof, the conflicting facts and the abductions that originated them are pruned. That means that at every point in time, the proof is consistent but defeasible. A broader and more detailed explanation of the abduction process including an example can be found at [11].

\section{RELATED WORK}

Many inteligent systems have been proposed in the last years. For instance, SUGGEST [14] and C-Graph [15] support user Web navigation dynamically generating links to pages that are unvisited by a user, and respectively monitoring user behavior and learning user preferences, to provide him with a set of recommendations. In the former historical information about the user behavior is maintained by means of an incremental graph partitioning algorithm, and in the latter the user knowledge is modeled into an ontology as a rooted labeled direct graph. CBCF [16] uses a content-based predictor to enhance existing user data, to exploit collaborative filtering so as to generate personalized suggestions. X-Compass [17] is an XML-based agent model that supports a user in his Web activities by monitoring

\footnotetext{
${ }^{3}$ That is, whenever a word that has not yet been queried in WordNet appears.
} 
the behavior in the Web pages access to automatically construct and manage his profile. X-Compass exploits such profiles to provide content-based and collaborative filtering recommendations. All the aforementioned systems exploit an internal profile to store information relative to the user. In that sense our approach is different because we do not need to define a model of the user as we exploit semantic relations based on runtime hypothesis creation.

\section{CONCLUSIONS AND FUTURE WORK}

In this paper we presented a system that can lead creative professionals on a individual brainstorm by using images to relate semantic concepts. We used an abduction reasoning process to infer some hypothesis about the user's ideation cognitive flow while it presents in an interactive way these inferences for appreciation.

As a next step we want to define a way for the described abductive relations to be reused in future interactions, driving this application to the semantic knowledge creation field, annotating new relations over WordNet graph that are not expressed on its lexical ground. For that, we will counter with a graph analysis system that detects possible cognitive similitudes between different users' creative flows and therefore use these users' past interactions with the system to enhance concept discover and context association. Future work also will focus on the integration of this architecture with a Multiagent System for non-symbolic fields, in those cases that deductive reasoning is not possible because of an incomplete knowledge about the state of the world.

Currently we have a prototype, accessible at http://tulum.lsi.upc.edu:8081/use, which is implemented as a Flash interface (see Figure 1). The underlying abduction process (see Figures 3, 4 and 5) is implemented in Ruby using the Ruby-WordNet and the Ruby-Flickr libraries.

\section{ACKNOWLEDGEMENTS}

S. Alvarez-Napagao, J. Vázquez-Salceda would like to acknowledge support from European funded CONTRACT Project (IST-034418). J. Sousa Lopes work has been funded by FCT-Portugal, scholarship SFRH/BD/38767/2007. J. Vázquez-Salceda work has been also partially funded by the Ramón y Cajal program of the Spanish Ministry of Education and Science. The content of this paper however solely reflects the opinion of the authors, and does not necessarily represent the views of the European Commission.

\section{REFERENCES}

[1] S. Card, T. Moran, and A. Newell, "The psychology of human-computer interaction," books.google.com, Jan 1983.

[2] M. Balabanović and Y. Shoham, "Fab: content-based, collaborative recommendation," Communications of the ACM, Jan 1997.
[3] G. Salton, "Automatic text processing," Addison-Wesley Longman Publishing Co., Jan 1988. [Online]. Available: http://portal.acm.org/citation.cfm?id=63601 dl=

[4] J. Breese, D. Heckerman, and C. Kadie, "Empirical analysis of predictive algorithms for collaborative filtering," Learning.

[5] G. Adomavicius and A. Tuzhilin, "Toward the next generation of recommender systems: A survey of the state-of-the-art and possible extensions," IEEE transactions on knowledge and data engineering, Jan 2005.

[6] C. Basu, H. Hirsh, and W. Cohen, "Recommendation as classification: Using social and contentbased information in recommendation," PROCEEDINGS OF THE NATIONAL CONFERENCE ON ARTIFICIAL INTELLIGENCE, Jan 1998. [Online]. Available: http://www.aaai.org/Papers/AAAI/1998/AAAI98-101.pdf

[7] R. Sinha, "A cognitive analysis of tagging," 2005. [Online]. Available: http://rashmisinha.com/2005/09/27/acognitive-analysis-of-tagging/

[8] S. Golder and B. Huberman, "The structure of collaborative tagging systems," Arxiv preprint cs.DL, Jan 2005. [Online]. Available: http://arxiv.org/abs/cs/0508082

[9] G. Miller, "Wordnet: a lexical database for english," Communications of the ACM, Jan 1995. [Online]. Available: http://portal.acm.org/citation.cfm?doid=219717.219748

[10] M. Sindlar, M. Dastani, F. Dignum, and J. Meyer, "Mental state abduction of bdi-based agents," Declarative Agent Languages and Technologies VI: 6th International Workshop on Declarative Agent Languages and Technologies, Jan 2009.

[11] J. S. Lopes, S. Alvarez-Napagao, S. Reis, and J. VazquezSalceda, "Reasoning about abductive inferences in bdi agents," Technical Report LSI-09-12-R, Universitat Politècnica de Catalunya, pp. 1-1, Apr 2009.

[12] U. Eco and T. Sebeok, "The sign of three," Indiana University Press, Jan 1983.

[13] J. Meheus and D. Batens, "A formal logic for abductive reasoning," Logic Journal of IGPL, Jan 2006. [Online]. Available: http://jigpal.oxfordjournals.org/cgi/content/abstract/14/2/221

[14] F. Silvestri, R. Baraglia, P. Palmerini, and M. Serranò, "Online generation of suggestions for web users," Information Technology: Coding and Computing, Jan 2004.

[15] F. Buccafurri, G. Lax, D. Rosaci, and D. Ursino, "A user behavior-based agent for improving web usage," LECTURE NOTES IN COMPUTER SCIENCE, Jan 2002.

[16] P. Melville, R. Mooney, and R. Nagarajan, "Content-boosted collaborative filtering for improved recommendations," PROCEEDINGS OF THE NATIONAL CONFERENCE ON ARTIFICIAL INTELLIGENCE, Jan 2002. [Online]. Available: https://www.aaai.org/Papers/AAAI/2002/AAAI02-029.pdf

[17] S. Garruzzo, S. Modafferi, D. Rosaci, and D. Ursino, "An xml-based agent model for supporting user activities on the web," Web Intelligence and Agent Systems, Jan 2006. 